\title{
A Comparative Study of the Three English Versions of Lines Sent to the North Written during Night Rains from the Perspective of Transitivity
}

\author{
Ningning Wang \\ Department of Foreign Languages, East China University of Science and Technology, Shanghai, China \\ Email: elainewang66@foxmail.com
}

How to cite this paper: Wang, N.N. (2020) A Comparative Study of the Three English Versions of Lines Sent to the North Written during Night Rains from the Perspective of Transitivity. Open Access Library Journal, 7: e6474.

https://doi.org/10.4236/oalib.1106474

Received: May 27, 2020

Accepted: June 19, 2020

Published: June 22, 2020

Copyright (๑) 2020 by author(s) and Open Access Library Inc.

This work is licensed under the Creative Commons Attribution International License (CC BY 4.0).

http://creativecommons.org/licenses/by/4.0/

\begin{abstract}
The poem Lines Sent to the North Written during Night Rains is one of the most famous poems of the late Tang poet Li Shangyin. It is widely favored by translators and has many English versions. Through the literature research, it is found that there are few studies of its English versions combined with the linguistic tool of transitivity. This paper aims to study the poem Lines Sent to the North Written during Night Rains and its three English versions from the perspective of system-functional linguistics, taking the concept of transitivity as a theoretical tool, supplemented by the theory of functional equivalence to evaluate the three English versions. According to the results of transitivity characteristic analysis and functional equivalence evaluation, it is found that keeping the transitivity characteristics of the original and the translation consistent is actually a kind of application and embodiment of the principle of functional equivalence, and the change of transitivity characteristics does not necessarily violate the principle of functional equivalence, because in specific circumstances, the transformation of process types can also help to achieve the effect of meaning equivalence, reproduce the artistic conception, and finally realize the goal of functional equivalence.
\end{abstract}

\section{Subject Areas}

Linguistics, Translation

\section{Keywords}

Transitivity, English Versions, Lines Sent to the North Written during Night Rains 


\section{Introduction}

With the wide application of system-functional linguistics, Chinese scholars have made many achievements in the study of transitivity and discourse analysis, such as the critical discourse analysis of news discourse based on various processes of transitivity system, the contrastive analysis of English and Chinese novel discourse from the perspective of transitivity, etc. While in the research direction of ancient poetry translation, most scholars widely use the perspective of "faithfulness, expressiveness and elegance" to compare and appreciate the translations. Professor Huang Guowen has selected six English versions of Qingming for the comparative study [1], and has successfully applied the system-functional linguistics to the study of poetry translation. Since then, scholars have also begun to study poetry translation from the perspective of transitivity, such as exploring the formal equivalence in the English translations of Song of a Roamer from the perspective of transitivity in system-functional linguistics [2], etc. In order to enrich the research field of comparative analysis of ancient poetry translation from the perspective of system-functional linguistics, this paper uses transitivity system as a theoretical tool, supplemented by the theory of functional equivalence as a criterion to evaluate the poetry translation, so as to objectively evaluate the effect of translation under the condition of equivalence and nonequivalence of transitivity characteristics between the translated text and the original text, and finally reveal the relationship between transitivity system and functional equivalence in translation.

\section{An Overview of Transitivity Theory}

System-functional linguistics explains language structure with language function and shows the idea that language has ideational function, interpersonal function and textual function [3]. The ideational function includes two parts: experiential function and logical function. Experiential function refers to the language expression of people's various experiences in the real world (including the inner world). In other words, it reflects the factors such as what happened in the objective and the subjective world, the people and things involved, and the time and place related to them. The experiential function is mainly embodied by transitivity and voice. Halliday believes that transitivity system divides what people see, hear and do in the real world into several processes and shows them in the clauses, including six categories: material process, mental process, relational process, behavioral process, verbal process and existential process (seeing Table 1) [4]. The transitivity system of language is constituted by these six processes, revolving around these processes are participants and circumstances, which should also be taken into consideration.

\section{The Significance of Transitivity System and Functional Equivalence Theory in Chinese Ancient Poetry Translation Evaluation}

The systemic functional grammar created and developed by Halliday provides a 
Table 1. The six process types of transitivity system.

\begin{tabular}{|c|c|c|c|}
\hline Process & Meaning & Participant & Example \\
\hline Material process & A process of doing & Actor-Goal & $\begin{array}{l}\text { My brother built all } \\
\text { these houses. }\end{array}$ \\
\hline Mental process & A process of thinking & Senser-Phenomenon & She likes the gift. \\
\hline \multirow{2}{*}{ Relational process } & \multirow{2}{*}{ A process of being } & Attributive: Carrier-Attribute & The cat is on the mat. \\
\hline & & Identifying: Identifier-Identified & Sarah is the wise one. \\
\hline Behavioral process & A process of behaving & Behaver & $\begin{array}{l}\text { He sighed for the day } \\
\text { of his youth. }\end{array}$ \\
\hline Verbal process & A process of saying & Sayer-Verbiage-Receiver & $\begin{array}{c}\text { She asked me a lot of } \\
\text { questions. }\end{array}$ \\
\hline Existential process & A process of existing & Existent & $\begin{array}{c}\text { There is a pen on the } \\
\text { desk. }\end{array}$ \\
\hline
\end{tabular}

new perspective for the evaluation and analysis of ancient poetry translation. The transitivity system in systemic functional grammar is a semantic system to express the ideational function, it is a systematic network about the process types, participants and circumstances [3]. The purpose of transitivity analysis is to analyze the experiential meaning of the text, and to find out the main content of the text by analyzing the types of process and participants [5]. Although there are plenty of translations of famous ancient poems, there are few studies on poetry translation evaluation from the perspective of transitivity. In order to enrich the research field of comparison and appreciation of ancient poetry translation from the perspective of system-functional linguistics, it is necessary to use transitivity system and functional equivalence theory to study poetry translation, so as to objectively evaluate the effect of translation under the condition of equivalence and nonequivalence of transitivity characteristics between the translated text and the original text, and finally explore the relationship between transitivity system and functional equivalence theory.

Eugene Nida has pointed out the different stages of the theory of functional equivalence: the low-level, realistic "functional equivalence" and the high-level, ideal "functional equivalence". Nida believes that the effect of translation can not only be judged by comparing the meaning, grammar and rhetoric of the translated text and the original text, but also by the target readers' correct understanding and appreciation of the translation [6]. Based on Nida's functional equivalence theory and the special literary genre of ancient poetry, it is easy to find that the translation of ancient poetry should be equivalent to the original in meaning, style and culture. Generally speaking, the realization of meaning equivalence refers to the selection of appropriate words to convey the meaning of the source language; the realization of style equivalence refers to the reproduction of the original images; the realization of cultural equivalence refers to the correct translation of allusions and the transmission of cultural information. These three standards can help to objectively compare and appreciate the English versions of ancient poems, make the translation quality evaluation of ancient poems more 
evidence-based, and help to comprehensively judge which translation can achieve the effect of making the target readers understand the original poems accurately.

\section{Lines Sent to the North Written during Night Rains and Its Three English Versions}

Li Shangyin is a famous Chinese poet in the late Tang Dynasty. As his representative lyric poem, Lines Sent to the North Written during Night Rains is full of touching emotional glamor. The original poem is as follows (with Pinyin):

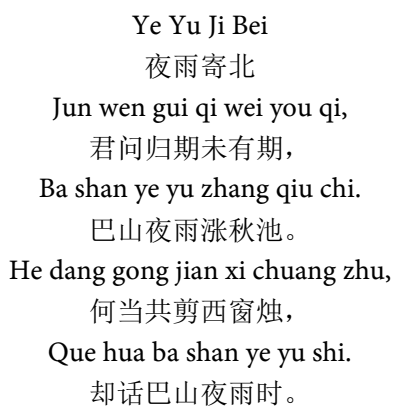

This poem is a seven-character quatrain (four-line poem with seven characters per line) written by Li Shangyin to his wife in Chang'an when he lived in Bashu (ancient cities in Sichuan). At that time, the poet was stuck in Bashu area because of the autumn rain. His wife sent letters from home to ask about his return date. The first two sentences of the poem, with the question and answer and the description of immediate environment, convey the poet's loneliness and deep concern for his wife. In the last two sentences, he imagined the joy of meeting again in the future, which serves as a foil to the loneliness of night. The meaning of the whole poem is: You ask me the date of my return, but my return date has not been set yet. Tonight's Mount $\mathrm{Pa}$, with continuous autumn rain, the pool is overfull with water. When can we trim a candlewick together near the west window, and tell the story of the night rain on Mount Pa today? The poem is improvised to express the poet's sudden mood changes. The language is simple, and there is no trace of modification in the choice of words and sentences. It is different from most of Li Shangyin's poems in terms of rhetoric and diction. In contrast, this poem is plain and natural, delicate in feelings, and graceful in artistic conception. Many translators and scholars have translated this famous poem of Li Shangyin, including the British Sinologist Herbert Allen Giles, the well-known Chinese litterateur Lin Yutang, and Xu Yuanchong, a famous translator of Chinese ancient poetry. They are highly-educated and keen on Chinese literature, while all have different specialized directions. What's more, because of the different nationality, Herbert Allen Giles may also provide some particular understanding and explanation of this poem, therefore, their three English versions have great significance in the comparative study of Chinese ancient poetry translations from the perspective of transitivity system. The three English ver- 
sions chosen as the research materials are as follows (seeing Table 2) [7].

\subsection{The Translation of “君问归期未有期”}

Version 1: You ask when I'm coming: alas not just yet...

Version 2: You ask how long before I come. Still no date is set.

Version 3: You ask me when I can return, but I don't know.

There are two verbal processes in the first sentence of version 1 . In this sentence, "alas not just yet..." is preceded by a colon, as if the poet is answering, which conforms to the "question and answer" in the original meaning. In addition, the sigh of "alas" shows the author's helplessness in not knowing the date of return, and the ellipsis at the end of the sentence indicates that the date of return is hard to be determined and far away, which better achieve the meaning equivalence with the original.

Version 2 translates the first sentence of the original text into two sentences, showing a verbal process and a material process, and the material process "still no date is set" presents an objective fact that the date of return is not determined, and also vividly shows the scene of "question and answer" between wife and husband. From the aesthetic point of view, it fully reflects the unity of poetry and artistic conception in the original text, and accurately conveys the connotation of "the date of return is difficult to be determined".

Version 3 translates the first sentence into a compound-complex sentence, which shows a verbal process and a mental process. Compared with version 1 and version 2, the word "return" better reflects the meaning of going back home. Besides, the mental process of "but I don't know" also succinctly and vividly conveys the helplessness of the poet when he was stuck in a place far away from home.

To sum up, version 1 is equivalent to the original text in transitivity characteristics, while version 2 and version 3 are not fully in line with the transitivity characteristics of the original poem, but all the three versions have achieved the goal of meaning equivalence. That is to say, the transformation of process types may not influence the effect of achieving meaning equivalence and the connotation of the source language can also be reproduced. Since the first sentence of the original poem does not contain the images and allusions, the style equiva-

Table 2. Three English versions of Lines Sent to the North Written during Night Rains.

\begin{tabular}{|c|c|c|}
\hline Herbert Allen Giles: Version 1 & Lin Yutang: Version 2 & Xu Yuanchong: Version 3 \\
\hline & & You ask me when I can return, but I don't \\
\hline You ask when I'm coming: alas not just yet... & You ask how long before I come. Still no date is set. & know; \\
\hline How the rain filled the pools on that night & The night rains on Mount Pa swell the autumn pool. & It rains in western hills and autumn pool \\
\hline when we met! & When shall we, side by side, trim a candle at the west & o'er-flow. \\
\hline Ah, when shall we ever snuff candles again, & window, & When can we trim by \\
\hline rain? & $\mathrm{Pa} ?$ & $\begin{array}{l}\text { And talk about the western hills in rainy } \\
\text { night? }\end{array}$ \\
\hline
\end{tabular}


lence and cultural equivalence between the translations and the original are not judged temporarily. It is easy to find that the three versions basically achieve the functional equivalence with the original text, which reflects that the change of transitivity characteristics does not necessarily violate the principle of functional equivalence.

\subsection{The Translation of “巴山夜雨涨秋池”}

Version 1: How the rain filled the pools on that night when we met!

Version 2: The night rains on Mount Pa swell the autumn pool.

Version 3: It rains in western hills and autumn pool o'er-flow.

In version 1, the exclamatory sentence started with "how" is used to show a mental process and a material process. In the literal sense, it means that "on that night when we met the heavy rain filled the pools", obviously, "filled" and "met" are in the past tense, and the misuse of tense causes the dislocation of time and space, which hides the poet's description of the scene before his eyes, thus destroys the artistic conception shown in the original text. However, if the translation is adjusted to "How the rain on Mount Pa fills the autumn pools tonight!", it will not only keep consistent with the participants and circumstantial elements of the original, but also retain the characteristic of the combination of emotion and scenery in the original text.

Version 2 takes “巴山夜雨” as a whole part and translates it into "the night rains on Mount Pa". In this sentence, the word "swell" not only expresses the meaning of "water level rises" in the original text, but also implies the poet's deep concern for his wife. What's more, every word in translation corresponds to the original text, without any additional translations or omissions. The original images of "the night rains on Mount Pa" and "the autumn pool" are completely displayed in front of the readers, which help to achieve the goal of style equivalence with the original, so that the readers can feel the artistic conception of the original text in a better way.

In version 3, the second sentence of the original text is separated into "it rains in western hills" and "autumn pool o'er-flow", which form two material processes. "It" is set as the subject of the impersonal verb, and juxtaposes the subject-predicate structures of "it rains" and "autumn pool o'er-flow". The word "o'er-flow" also well embodies the feature of the original poem, which turns the poet's deep concern for his wife into an overflowing pool. “巴山” originally refers to the Daba Mountain (at the junction of the south of Shaanxi and the northeast of Sichuan), and in the old times, it refers to a very remote place. Here, the word "巴山” is translated into "western hills". Although it is easy to be understood in geographical position, it may be confused with “西山 (western hills in the literal sense)" in place name. "巴山" has the implication of being relegated to a desolate place. Although this implication is not easy to be embodied in the translation, the literal translation method is a more practical way to reflect this place name. 
It is easy to find that version 2 is better than version 1 and version 3 in terms of meaning equivalence, and because of the accurate grasp of images and the literal translation of the word “巴山”, version 2 is also better in terms of style equivalence and cultural equivalence with the original text. It can be seen that if the circumstantial elements such as place and time contained in the original text are omitted or mistranslated, it may influence the effect of functional equivalence between the translated text and the original text. Therefore, it can be concluded that keeping the transitivity characteristics of the original text (including the participants and circumstantial elements) in the translation is helpful to reproduce the artistic conception of the source language and achieve the goal of functional equivalence.

\subsection{The Translation of “何当共剪西窗烛”}

Version 1: Ah, when shall we ever snuff candles again.

Version 2: When shall we, side by side, trim a candle at the west window.

Version 3: When can we trim by window side the candlelight.

Version 1 is roughly equivalent to the original poem in terms of transitivity characteristics, but it lacks a place element“西窗 (the west window)”, which causes the inadequacy in circumstantial elements and a lack of background to set off the emotion by contrast. In terms of the translation of “剪”, “snuff” is “to extinguish a light from a naked flame, especially a candle”. In traditional Chinese culture, the word “剪烛” means “cut off the extra wick to maintain the bright lighting", and then this behavior is set as the allusion of having a heart to heart night talk. In this aspect, the meaning of "snuff candles" is quite different from that of the word “剪烛”. From the perspective of functional equivalence, it does not achieve the goal of cultural equivalence with the original poem, which causes the inadequacy of the original artistic conception.

Version 2 is almost equivalent to the original poem in transitivity characteristics, and takes "side by side" as the parenthesis to fully express the meaning of the character “共 (get together)" in the original poem. The word “剪烛” in this sentence is translated into "trim a candle", which is not in line with the original meaning of "trim the extra wick of candle". In this aspect, the cultural equivalence is not well achieved. By contrast, using the phrase "trim the candlewick" to express the original meaning is more appropriate.

Similar to version 2, the meaning of "trim the candlelight" in version 3 is not equivalent to the meaning of “剪烛” in the original text. What's more, "by window side" means "by the window", while the explanation of the location of window has not been reflected in the translation. In traditional Chinese literature, "west window", as a unique literary image, is just like the phrase "west wind". In "east, south, west, and north" and "spring, summer, autumn, and winter", the contrast between "west" and "autumn" reflects a feeling of sadness. In order to express the sorrow of separation, Chinese poets often use the image of "west window" to set off the emotion by contrast. However, in this translation, there is 
a lack of artistic conception reproduction, which results in the lack of style equivalence between the translation and the original.

To sum up, from the perspective of process type of transitivity, all the three versions are consistent with the original text. However, in the aspect of participant's confirmation, for the word “剪烛”, all the three translators have not provided a translation that conforms to the cultural allusion contained in the original text, so their translations have not achieved the goal of cultural equivalence with the original. What's more, in the translation of the word “西窗”, version 1 and version 3 have different degrees of omissions, which result in the lack of artistic conception conveyed by the image of "west window", and also fail to achieve the meaning and style equivalence with the original poem. Obviously, there is a positive correlation between transitivity system and translation functional equivalence. Keeping the transitivity characteristics of the translation and the original consistent is actually an application and embodiment of the principle of functional equivalence, which is helpful to make the structure and expression effect of the translation similar to the original. In other words, it will result in the differences between the transitivity characteristics of the translation and the original if the cultural background of the original text is not fully understood, and ultimately the goal of functional equivalence between the translation and the original text cannot be achieved.

\subsection{The Translation of “却话巴山夜雨时”}

Version 1: And recall the glad hours of that evening of rain?

Version 2: And (we) talk to the time of the night rains on Mount $\mathrm{Pa}$ ?

Version 3: And talk about the western hills in rainy night?

Version 1 translates “却话” into "recall”, which shows a mental process. In the original text, the meaning of the word “却话” is “tell about the past", which shows a verbal process. Although version 1 is not consistent with the original text in the process type, it can better achieve the meaning equivalence with the original when it is unable to find the corresponding word in English. However, the translation "recall the glad hours of that evening of rain" deviates from the original meaning. In the last two lines of the poem, the poet envisages the future reunion. On the day of meeting, the poet will tell the sadness of departure of that night again, which is contrasted with the joy of imagining the future reunion. However, this translation recalls the past, and once again causes the dislocation of time and space, which results in the disappearance of the artistic conception created by the contrast of the time and space in the original text.

In version 2, the sentence “巴山夜雨时” is translated into “the time of the night rains on Mount $\mathrm{Pa}$ ". In this translation, each word corresponds to the original text, which reproduces the original image without any additional translations or omissions, and achieves the goal of meaning equivalence and style equivalence with the original text. However, the phrase "talk to" means "speak to (someone)", while in the translation, the phrase "talk to" is connected with a 
noun phrase, which is not in line with English grammar.

Version 3 is basically equivalent to the original in transitivity characteristics, while in the translation of “却话”, the phrase “talk about” seems to refer to a usual behavior. Due to the meaning of "tell about the past" in the original text, in contrast, the word "recall" can better convey the original meaning.

To sum up, compared with the word “却话” in the original text, the use of "recall" in version 1 makes the process type change from verbal process to mental process, but it better conveys the meaning of "tell about the past" in the original text and achieves the goal of meaning equivalence. Take version 2 as an example, if replace "talk to" with "recall", that is, "And (we) recall the time of the night rains on Mount $\mathrm{Pa}$ ?", then the translation will basically achieve the functional equivalence with the original. On the whole, version 2 does better in meaning equivalence, style equivalence and cultural equivalence with the original text. This part of the analysis once again reflects that the change of transitivity characteristics does not necessarily violate the principle of functional equivalence, because in specific circumstances, only through the transformation of process types can the translator better reflect the differences between the source language and the target language, and better express the artistic conception of the source language.

\section{Conclusion}

Based on the above study of transitivity and functional equivalence, it is found that each of these translations has its own advantages and disadvantages. Compared with the translators using target language as the first language, the translators using source language as the first language can better understand the meaning, artistic conception and emotion of the original text. In the comparison of these three English versions, it is found that Lin Yutang's translation is slightly better than the others. His translation not only keeps the transitivity characteristics consistent with the original for the most part, but also faithfully conveys the core connotation of the original poem, and basically achieves the effect of meaning equivalence, style equivalence and cultural equivalence. In the process of comparing the results of transitivity characteristic analysis and functional equivalence evaluation, it is found that keeping the transitivity characteristics of the translation and the original consistent is actually an application and embodiment of the principle of functional equivalence, which makes the structure and expression effect of the translation similar to the original. Although the deviations in translation caused by the differences between the source language and the target language still exist, this kind of influence can be offset by the full understanding of the original text and the accurate expression of the translation. What's more, the change of transitivity characteristics does not necessarily violate the principle of functional equivalence, because in specific circumstances, the transformation of process types can also help to achieve the effect of meaning equivalence, reproduce the artistic conception, and finally achieve the goal of 
functional equivalence. This paper makes a comparative study of the three English versions of Lines Sent to the North Written during Night Rains from the limited perspective of transitivity, and explores the relationship between transitivity system and functional equivalence. In the field of translation study from the perspective of system-functional linguistics, it is still a preliminary attempt. In the future, it is meaningful to make further research on the translation of ancient poetry from the perspectives of interpersonal function and textual function in system-functional linguistics, so as to expand the effective means of evaluating the translation quality of Chinese ancient poetry.

\section{Conflicts of Interest}

The author declares no conflicts of interest regarding the publication of this paper.

\section{References}

[1] 黄国文. 《清明》一诗英译文的人际功能探讨 [J]. 外语教学, 2002, 23(3): 34-38.

[2] 张威. 功能语言学关照下的翻译形式等效——《游子吟》英译文的及物性剖析 [J]. 当代外语研究, 2010(5): 18-21.

[3] 何伟, 魏榕. 系统功能语言学及物性理论发展综述[J]. 北京科技大学学报, 2016, 32(1): 1-20.

[4] 胡壮麟, 朱永生, 张德禄, 李战子. 系统功能语言学概论 $[\mathrm{M}]$. 北京: 北京大学出 版社, 2005.

[5] 胡壮麟, 朱永生, 张德禄. 系统功能语法概论 [M]. 湖南: 湖南教育出版社, 1989.

[6] Nida, E. (1993) Language, Culture, and Translating. Shanghai Foreign Language Education Press, Shanghai.

[7] 刘国忠. 意境与古诗英译一一评李商隐《夜雨寄北》的三种译文[J]. 黄山学院学 报, 2011, 13(2): 86-89. 